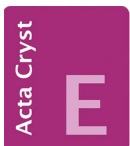

CRYSTALLOGRAPHIC COMMUNICATIONS

ISSN 2056-9890

Received 28 August 2019

Accepted 18 September 2019

Edited by B. Therrien, University of Neuchâtel, Switzerland

Keywords: crystal structure; Hofmann clathrate; iron(II).

Supporting information: this article has supporting information at journals.iucr.org/e

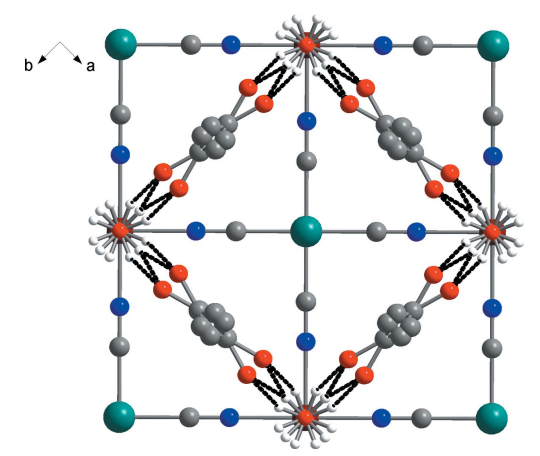

OPEN $\partial$ ACCESS

\section{Crystal structure of poly[[diaquatetra- $\mu_{2}$-cyanido- iron(II)platinum(II)] acetone disolvate]}

\author{
Iryna S. Kuzevanova, ${ }^{\mathrm{a}, \mathrm{b} *}$ Dina D. Naumova, ${ }^{\mathrm{b}}$ Kateryna V. Terebilenko, ${ }^{\mathrm{b}}$ Sergiu \\ Shova ${ }^{c}$ and II'ya A. Gural'skiy,d
}

\begin{abstract}
${ }^{a}$ Department of General and Inorganic Chemistry, National Technical University of Ukraine "Igor Sikorsky Kyiv Polytechnic Institute", Prosp. Peremogy 37, Kyiv 03056, Ukraine, 'bepartment of Chemistry, Taras Shevchenko National University of Kyiv, Volodymyrska St. 64, Kyiv 01601, Ukraine, 'Department of Inorganic Polymers, "Petru Poni" Institute of Macromolecular Chemistry, Romanian Academy of Science, Aleea Grigore Ghica Voda 41-A, lasi 700487, Romania,

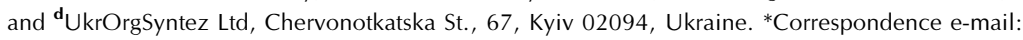
iryna.kuzevanova@univ.kiev.ua
\end{abstract}

In the title polymeric complex, $\left\{\left[\mathrm{FePt}(\mathrm{CN})_{4}\left(\mathrm{H}_{2} \mathrm{O}\right)_{2}\right] \cdot 2 \mathrm{C}_{3} \mathrm{H}_{6} \mathrm{O}\right\}_{n}$, the $\mathrm{Fe}^{\mathrm{II}}$ cation has an octahedral $\left[\mathrm{FeN}_{4} \mathrm{O}_{2}\right]$ geometry being coordinated by two water molecules and four cyanide anions. The Pt cation is located on an inversion centre and has a square-planar coordination environment formed by four cyanide groups. The tetracyanoplatinate anions bridge the $\mathrm{Fe}^{\mathrm{II}}$ cations to form infinite twodimensional layers that propagate in the $b c$ plane. Two guest molecules of acetone per $\mathrm{Fe}^{\mathrm{II}}$ are located between the layers. These guest acetone molecules interact with the coordinated water molecules by $\mathrm{O}-\mathrm{H} \cdots \mathrm{O}$ hydrogen bonds.

\section{Chemical context}

Hofmann clathrates and their analogues form one the most famous families of compounds that are able to incorporate guest molecules. The first clathrate was obtained by Hofmann and Küspert in 1897 (Hofmann \& Küspert, 1897) and was of composition $\left[\mathrm{Ni}\left(\mathrm{NH}_{3}\right)_{2} \mathrm{Ni}(\mathrm{CN})_{4}\right] \cdot 2 \mathrm{C}_{6} \mathrm{H}_{6}$. It was a $2 \mathrm{D}$ coordination compound formed by infinite cyanometallic layers that propagate along the $a b$ plane. The 2D system was supported by ammine axial ligands, and guest molecules of benzene were trapped between the layers.

Later, by slight modifications of the chemical composition, several analogous compounds were obtained, leading to the creation of a new class of coordination materials. The first modification was the substitution of nickel with other transition metals, as well as the introduction of other small aromatic guest molecules that resulted in the creation of compounds with the general formula $\left[M\left(\mathrm{NH}_{3}\right)_{2} M^{\prime}(\mathrm{CN})_{4}\right] \cdot 2 G$ (where $M=$ $\mathrm{Mn}, \mathrm{Fe}, \mathrm{Co}, \mathrm{Ni}, \mathrm{Cu}, \mathrm{Zn}, \mathrm{Cd}, M^{\prime}=\mathrm{Ni}, \mathrm{Pd}, \mathrm{Pt}$ and $G=$ benzene, pyrrole, thiophene, aniline, biphenyl, etc.; Iwamoto, 1996). The second modification of the Hofmann clathrate was the substitution of square-planar $\left\{M^{\prime}(\mathrm{CN})_{4}\right\}^{2-}$ anions with tetrahedral $\left(\left\{M^{\prime}(\mathrm{CN})_{4}\right\}^{2-}, M^{\prime}=\mathrm{Cd}, \mathrm{Hg}\right.$; Arcís-Castillo et al., 2013), linear $\left(\left\{M^{\prime}(\mathrm{CN})_{2}\right\}^{-}, M^{\prime}=\mathrm{Cu}, \mathrm{Ag}, \mathrm{Au}\right.$; Gural'skiy, Golub et al., 2016; Gural'skiy, Shylin et al., 2016), octahedral $\left(\left\{M^{\prime}(\mathrm{CN})_{6}\right\}^{3-}\right.$, $M^{\prime}=$ Co, Cr; Dommann et al., 1990) and even dodecahedral $\left(\left\{M^{\prime}(\mathrm{CN})_{8}\right\}^{4-}, M^{\prime}=\mathrm{W}, \mathrm{Nb}\right.$; Ohkoshi et al., 2013) fragments. Additionally, a very important modification was made by the introduction of other organic ligands instead of ammonia. For example, by the introduction of pyridine, the first $\mathrm{Fe}^{\mathrm{II}}$-based clathrate $\left[\mathrm{Fe}(\mathrm{py})_{2}\left\{\mathrm{Pt}(\mathrm{CN})_{4}\right\}\right]$ exhibiting spin-crossover (SCO) behaviour was obtained by Kitazawa et al. (1996). At the same 
time, the introduction of various bidentate ligands such as pyrazine (Niel et al., 2001; Gural'skiy, Shylin et al., 2016), pyrimidine (Agustí et al., 2008), bis(4-pyridyl)acetylene (Agustí et al., 2008) and others allowed the formation of 3D SCO networks.

Additionally, the characteristics of spin transition in coordination compounds are known to be extremely sensitive to any changes in the chemical environment. As Hofmann clathrate analogues are very easy to modulate, numerous SCO complexes with very different temperatures, abruptnesses and hystereses of SCO were obtained. Moreover, the ability of Hofmann clathrate analogues to incorporate guest molecules provided SCO-based chemical sensors (Ohba et al., 2009).

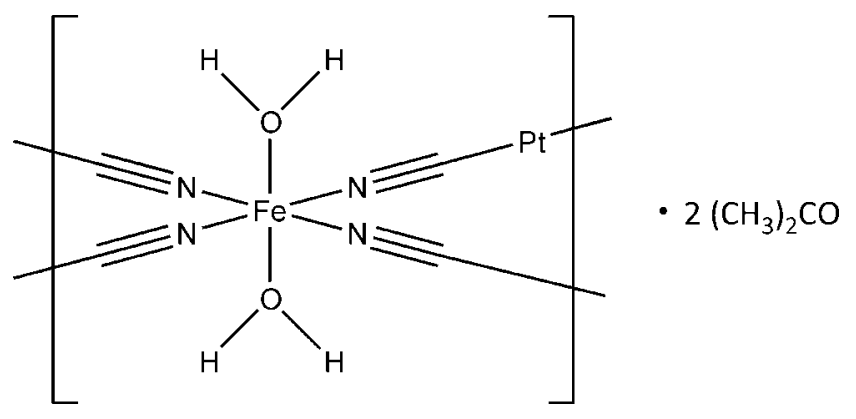

Herein we present a new Fe-Pt Hofmann clathrate analogue $\left[\mathrm{Fe}\left(\mathrm{H}_{2} \mathrm{O}\right)_{2}\left\{\mathrm{Pt}(\mathrm{CN})_{4}\right\}\right] \cdot 2\left(\mathrm{CH}_{3}\right)_{2} \mathrm{CO}$.

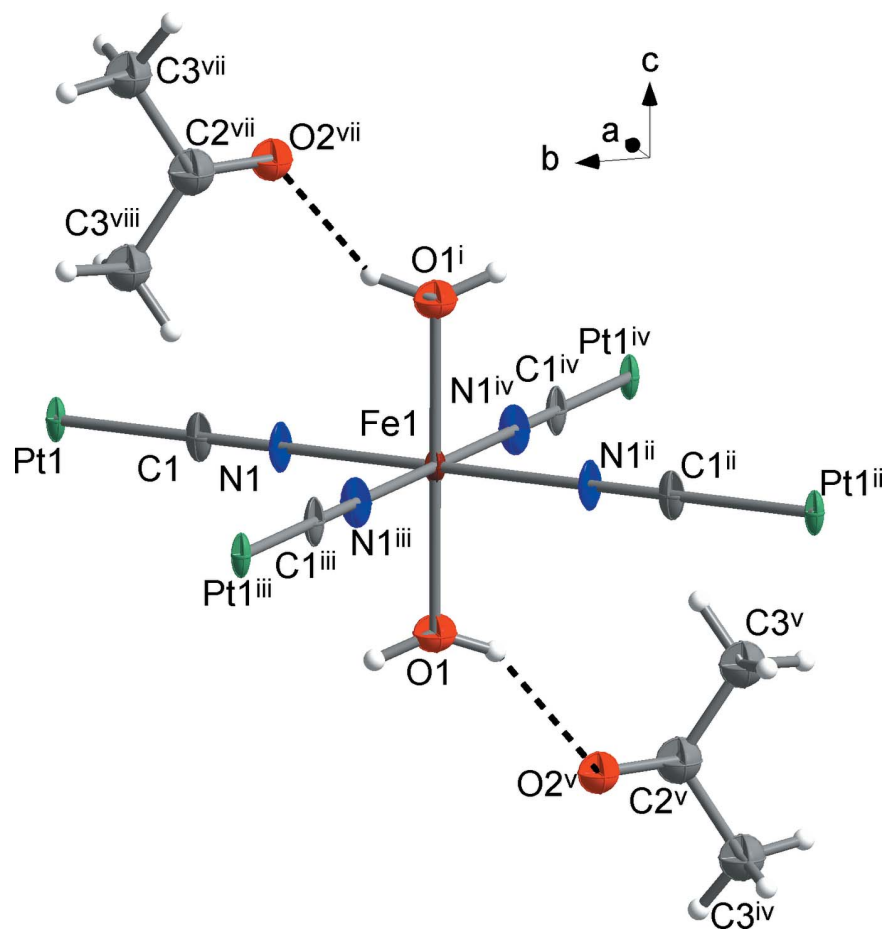

Figure 1

A fragment of the molecular structure of the title compound showing the atom-labelling scheme. Displacement ellipsoids are drawn at the $50 \%$ probability level [symmetry codes: (i) $x,-y, 1-z$; (ii) $-x,-y, z$; (iii) $x$, $-y, z$; (iv) $-x, y, z$; (v) $-1+x,-y, z$; (vi) $-1+x,-y,-z$; (vii) $1-x, y$, $1+z ;$ (viii) $1-x, y, 1-z]$.

\section{Structural commentary}

The title compound crystallizes in the $P 4 / \mathrm{mmm}$ space group. The $\mathrm{Fe}^{\mathrm{II}}$ cation has a $\left[\mathrm{FeN}_{4} \mathrm{O}_{2}\right]$ coordination environment (Fig. 1) comprising four $\mathrm{CN}^{-}$anions in the equatorial positions $[\mathrm{Fe} 1-\mathrm{N} 1=2.158$ (5) $\AA$ ] and two water molecules in the axial positions $[\mathrm{Fe} 1-\mathrm{O} 1=2.130$ (6) $\AA$ ] . The $\mathrm{Fe}-\mathrm{O}$ bonds are slightly shorter than the $\mathrm{Fe}-\mathrm{N}$ bonds, thus leading to a compressed octahedral geometry. Judging by the bond length, the $\mathrm{Fe}^{\mathrm{II}}$ cation is in a high-spin state at the experimental temperature $(180 \mathrm{~K})$. This is corroborated by the presence of $\mathrm{H}_{2} \mathrm{O}$ molecules in the coordination sphere of $\mathrm{Fe}^{\mathrm{II}}$. The cyanide anions connect the $\mathrm{Fe}^{\mathrm{II}}$ and $\mathrm{Pt}^{\mathrm{II}}$ cations into infinite twodimensional layers. The $\mathrm{Pt}^{\mathrm{II}}$ cation is located at a fourfold rotation axis and possesses a square-planar geometry [Pt1$\mathrm{C} 1=1.993$ (6) $\AA, \mathrm{C} 1-\mathrm{Pt}-\mathrm{C} 1=90^{\circ}$ ]. Thanks to the tetragonal symmetry of the crystalline compound, no deviation from an ideal octahedron is observed for $\mathrm{Fe}^{\mathrm{II}}, \Sigma|90-\theta|=0^{\circ}$, where $\theta$ is the $\mathrm{N}-\mathrm{Fe}-\mathrm{N}$ or $\mathrm{O}-\mathrm{Fe}-\mathrm{N}$ angles. Additionally, the compound incorporates two guest molecules of acetone per $\mathrm{Fe}^{\mathrm{II}}$ centers.

\section{Supramolecular features}

The crystalline structure is connected by bridging tetracyanoplatinate moieties, which form a two-dimensional grid that propagates along the $a b$ plane (Fig. 2). As imposed by

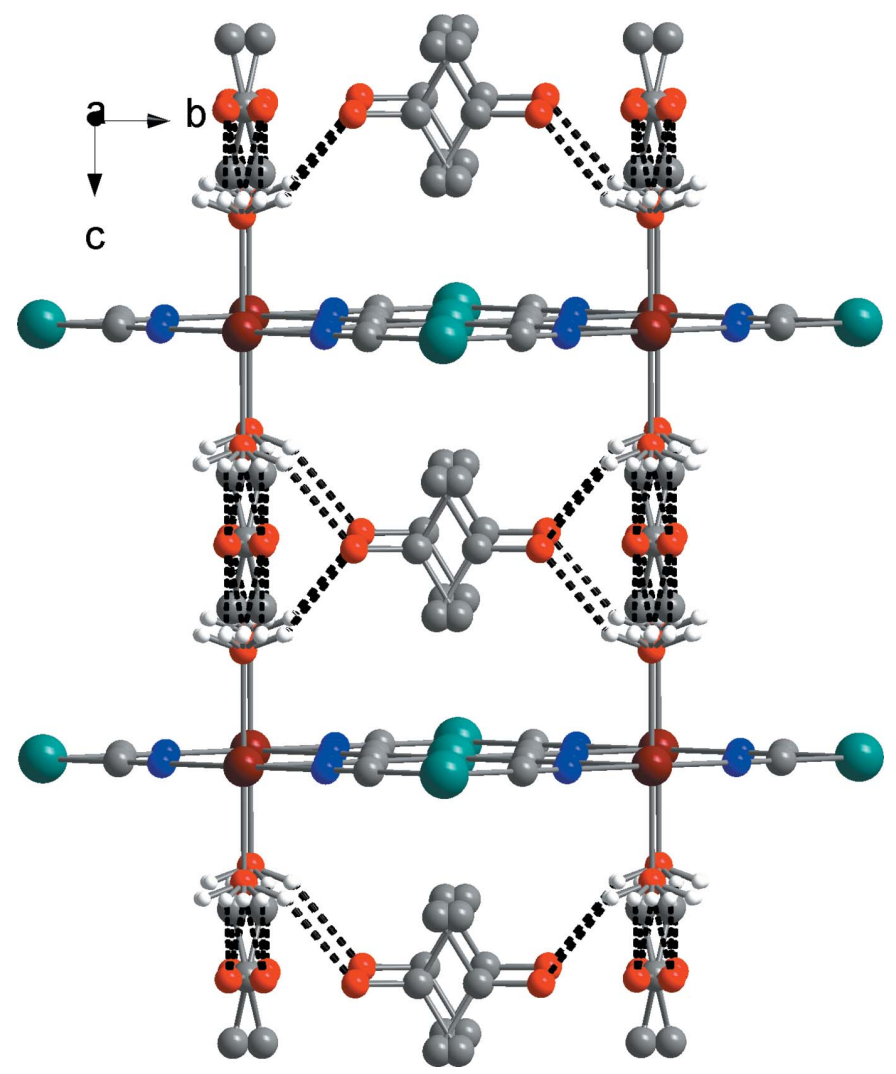

Figure 2

View of the crystal structure of the title compound in the $b c$ plane showing the two-dimensional cyanometallic layers. Hydrogen bonds are shown as dashed lines. Acetone $\mathrm{H}$ atoms are omitted for clarity. 
Table 1

Hydrogen-bond geometry $\left(\AA,^{\circ}\right)$.

\begin{tabular}{lllll}
\hline$D-\mathrm{H} \cdots A$ & $D-\mathrm{H}$ & $\mathrm{H} \cdots A$ & $D \cdots A$ & $D-\mathrm{H} \cdots A$ \\
\hline $\mathrm{O} 1-\mathrm{H} 1 A \cdots \mathrm{O} 2^{\mathrm{i}}$ & 0.84 & 2.03 & $2.775(11)$ & 147 \\
$\mathrm{O} 1-\mathrm{H} 1 A \cdots 2^{\mathrm{ii}}$ & 0.84 & 2.03 & $2.775(11)$ & 148 \\
$\mathrm{O} 1-\mathrm{H} 1 B \cdots \mathrm{O} 2^{\text {iii }}$ & 0.85 & 2.04 & $2.775(11)$ & 144 \\
$\mathrm{O} 1-\mathrm{H} 1 B \cdots \mathrm{O} 2^{\text {iv }}$ & 0.85 & 2.14 & $2.775(11)$ & 131 \\
\hline
\end{tabular}

Symmetry codes: (i) $-x+1,-y, z$; (ii) $-x+1, y,-z$; (iii) $-y,-x+1,-z$; (iv) $y,-x+1, z$.

symmetry, no deviation from linearity for the $\mathrm{Fe}-\mathrm{N}-\mathrm{C}-\mathrm{Pt}$ linkages is observed $\left[\mathrm{Fe}-\mathrm{N}-\mathrm{C}=180^{\circ}, \mathrm{N}-\mathrm{C}-\mathrm{Pt}=180^{\circ}, \mathrm{C}-\right.$ $\mathrm{Pt}-\mathrm{C}=180^{\circ}$. The distance between parallel cyanometallic layers is 7.973 (6) $\AA$. The guest acetone molecules are located between the cyanometallic layers. Each oxygen atom of the coordinated water molecules interacts with acetone by $\mathrm{O}-$ $\mathrm{H}$... O hydrogen bonds (Fig. 3, Table 1), creating a threedimensional supramolecular framewor. The size of the available voids between the cyanometallic layers allows the acetone molecules to rotate freely, thus leading to disorder of the acetone molecules over four positions.

\section{Database survey}

A survey of the Cambridge Structural Database (Version 5.38; Groom et al., 2016) confirmed that the title compound has never been published before. It revealed 51 cyanometallic structures of the general formula $\left[T M\left(\mathrm{H}_{2} \mathrm{O}\right)_{2}\left\{\mathrm{TM}(\mathrm{CN})_{4}\right\}\right]$, where $T M=$ any transition metal. There were also 19 hits for structures containing $\left[\mathrm{Fe}\left\{\mathrm{Pt}(\mathrm{CN})_{4}\right\}\right]$ fragments: refcodes: OVILEM, OVIRUI, OVIRUI01, OVIRUI02 and OVIRUI03 (Sciortino et al., 2017), AMIJOX (Kucheriv et al., 2016), BEDWEO and BEDWIS (Sciortino et al., 2012), CEMYUQ

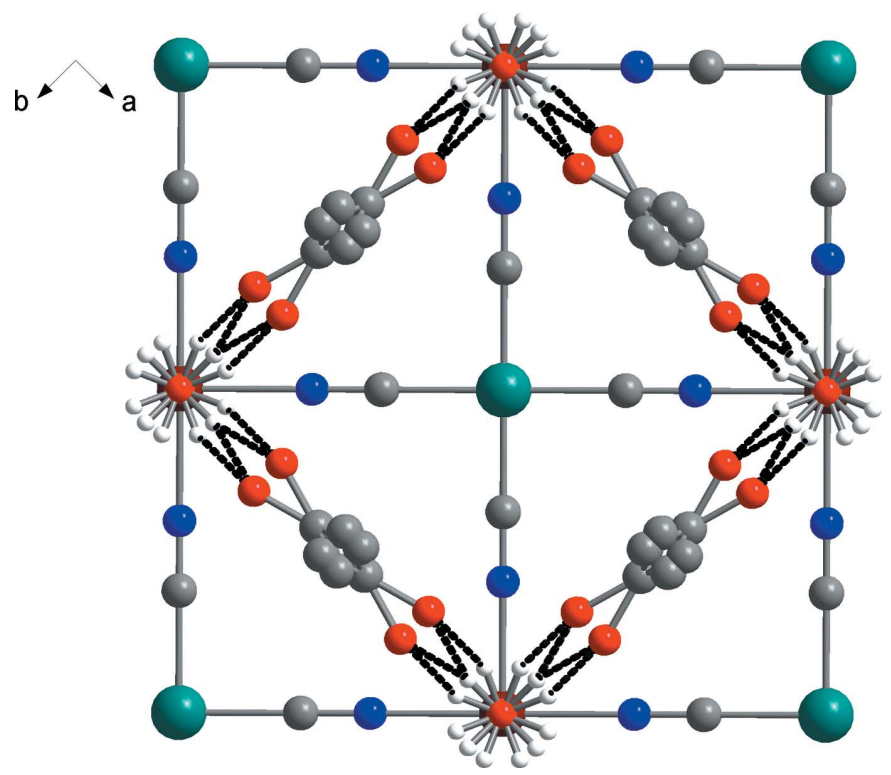

Figure 3

View of the structure of the title compound in the $a b$ plane showing the distortion of the acetone guest molecules. Hydrogen bonds are shown as dashed lines. Acetone $\mathrm{H}$ atoms are omitted for clarity.
Table 2

Experimental details.

\begin{tabular}{|c|c|}
\hline \multicolumn{2}{|l|}{ Crystal data } \\
\hline Chemical formula & {$\left[\mathrm{FePt}(\mathrm{CN})_{4}\left(\mathrm{H}_{2} \mathrm{O}\right)_{2}\right] \cdot 2 \mathrm{C}_{3} \mathrm{H}_{6} \mathrm{O}$} \\
\hline$M_{\mathrm{r}}$ & 507.21 \\
\hline Crystal system, space group & Tetragonal, $P 4 / \mathrm{mmm}$ \\
\hline Temperature (K) & 180 \\
\hline$a, c(\AA)$ & $7.4802(4), 7.9725(11)$ \\
\hline$V\left(\AA^{3}\right)$ & $446.09(8)$ \\
\hline$Z$ & 1 \\
\hline Radiation type & Mo $K \alpha$ \\
\hline$\mu\left(\mathrm{mm}^{-1}\right)$ & 8.66 \\
\hline Crystal size $(\mathrm{mm})$ & $0.05 \times 0.05 \times 0.02$ \\
\hline \multicolumn{2}{|l|}{ Data collection } \\
\hline Diffractometer & $\begin{array}{l}\text { Rigaku Oxford Diffraction } \\
\text { Xcalibur, Eos }\end{array}$ \\
\hline Absorption correction & $\begin{array}{l}\text { Multi-scan (CrysAlis PRO; Rigaku } \\
\text { OD, 2015) }\end{array}$ \\
\hline$T_{\min }, T_{\max }$ & $0.699,1.000$ \\
\hline $\begin{array}{l}\text { No. of measured, independent and } \\
\text { observed }[I>2 \sigma(I)] \text { reflections }\end{array}$ & $1126,361,359$ \\
\hline$R_{\text {int }}$ & 0.037 \\
\hline$(\sin \theta / \lambda)_{\max }\left(\AA^{-1}\right)$ & 0.682 \\
\hline \multicolumn{2}{|l|}{ Refinement } \\
\hline$R\left[F^{2}>2 \sigma\left(F^{2}\right)\right], w R\left(F^{2}\right), S$ & $0.025,0.046,1.04$ \\
\hline No. of reflections & 361 \\
\hline No. of parameters & 34 \\
\hline $\mathrm{H}$-atom treatment & $\mathrm{H}$-atom parameters constrained \\
\hline$\Delta \rho_{\max }, \Delta \rho_{\min }\left(\mathrm{e} \AA^{-3}\right)$ & $1.25,-1.06$ \\
\hline
\end{tabular}

Computer programs: CrysAlis PRO (Rigaku OD, 2015), SHELXT (Sheldrick, 2015a), SHELXL (Sheldrick, 2015b) and OLEX2 (Dolomanov et al., 2009).

(Mũnoz-Lara et al., 2013), MUHMEI, MUHNAF, MUHNAF01, MUHNAF02, MUHPAH and MUHPAH01 (Martínez et al., 2009), QADDUX (Sakaida et al., 2016), QOJWIW and QOJWIW01 (Cobo et al., 2008) and TURXIP (Ohtani et al., 2013).

\section{Synthesis and crystallization}

Crystals of the title compound were obtained by slow diffusion (three layers) in a $3 \mathrm{ml}$ tube. The first layer contained $19 \mathrm{mg}$ $(0.05 \mathrm{mmol})$ of $\mathrm{K}_{2}\left[\mathrm{Pt}(\mathrm{CN})_{4}\right]$ in $0.5 \mathrm{ml}$ of water. The middle layer contained $1.5 \mathrm{ml}$ of a water:acetone (1:1) solution. The third layer contained $25 \mathrm{mg}(0.05 \mathrm{mmol})$ of $\mathrm{Fe}(\mathrm{OTs})_{2} \cdot 6 \mathrm{H}_{2} \mathrm{O}$ in $0.4 \mathrm{ml}$ of acetone and $0.1 \mathrm{ml}$ of water. The colourless crystals grew in the middle layer within three weeks and were kept in the mother solution prior to measurements.

\section{Refinement}

Crystal data, data collection and structure refinement details are summarized in Table 2. All hydrogen atoms were fixed at calculated positions and refined as riding with $\mathrm{C}-\mathrm{H}=0.96 \AA$ and $\mathrm{O}-\mathrm{H}=0.84 \AA$, $U_{\text {iso }}(\mathrm{H})=1.5 U_{\text {iso }}(\mathrm{C}, \mathrm{O})$. The $\mathrm{OH}$ group and the idealized methyl group were refined as rotating.

\section{Funding information}

Funding for this research was provided by: Ministry of Education and Science of Ukraine (grant No. 19BF037-01M; grant No. 19BF037-01). 


\section{References}

Agustí, G., Thompson, A. L., Gaspar, A. B., Muñoz, M. C., Goeta, A. E., Rodríguez-Velamazán, J. A., Castro, M., Burriel, R. \& Real, J. A. (2008). Dalton Trans. pp. 642-649.

Arcís-Castillo, Z., Muñoz, M. C., Molnár, G., Bousseksou, A. \& Real, J. A. (2013). Chem. Eur. J. 19, 6851-6861.

Cobo, S., Ostrovskii, D., Bonhommeau, S., Vendier, L., Molnár, G., Salmon, L., Tanaka, K. \& Bousseksou, A. (2008). J. Am. Chem. Soc. 130, 9019-9024.

Dolomanov, O. V., Bourhis, L. J., Gildea, R. J., Howard, J. A. K. \& Puschmann, H. (2009). J. Appl. Cryst. 42, 339-341.

Dommann, A., Vetsch, H. \& Hulliger, F. (1990). Acta Cryst. C46, 1994-1996.

Groom, C. R., Bruno, I. J., Lightfoot, M. P. \& Ward, S. C. (2016). Acta Cryst. B72, 171-179.

Gural'skiy, I. A., Golub, B. O., Shylin, S. I., Ksenofontov, V., Shepherd, H. J., Raithby, P. R., Tremel, W. \& Fritsky, I. O. (2016). Eur. J. Inorg. Chem. 2016, 3191-3195.

Gural'skiy, I. A., Shylin, S. I., Golub, B. O., Ksenofontov, V., Fritsky, I. O. \& Tremel, W. (2016). New J. Chem. 40, 9012-9016.

Hofmann, K. A. \& Küspert, F. (1897). Z. Anorg. Chem. 15, 204-207. Iwamoto, T. (1996). J. Incl Phenom. Macrocycl Chem. 24, 61-132.

Kitazawa, T., Gomi, Y., Takahashi, M., Takeda, M., Enomoto, M., Miyazaki, A. \& Enoki, T. (1996). J. Mater. Chem. 6, 119-121.

Kucheriv, O. I., Shylin, S. I., Ksenofontov, V., Dechert, S., Haukka, M., Fritsky, I. O. \& Gural'skiy, I. A. (2016). Inorg. Chem. 55, 4906-4914.
Martínez, V., Gaspar, A. B., Muñoz, M. C., Bukin, G. V., Levchenko, G. \& Real, J. A. (2009). Chem. Eur. J. 15, 10960-10971.

Muñoz-Lara, F. J., Gaspar, A. B., Muñoz, M. C., Ksenofontov, V. \& Real, J. A. (2013). Inorg. Chem. 52, 3-5.

Niel, V., Martinez-Agudo, J. M., Muñoz, M. C., Gaspar, A. B. \& Real, J. A. (2001). Inorg. Chem. 40, 3838-3839.

Ohba, M., Yoneda, K., Agustí, G., Muñoz, M. C., Gaspar, A. B., Real, J. A., Yamasaki, M., Ando, H., Nakao, Y., Sakaki, S. \& Kitagawa, S. (2009). Angew. Chem. Int. Ed. 48, 4767-4771.

Ohkoshi, S., Takano, S., Imoto, K., Yoshikiyo, M., Namai, A. \& Tokoro, H. (2013). Nat. Photonics. 8, 65-71.

Ohtani, R., Arai, M., Ohba, H., Hori, A., Takata, M., Kitagawa, S. \& Ohba, M. (2013). Eur. J. Inorg. Chem. 2013, 738-744.

Rigaku OD (2015). CrysAlis PRO. Rigaku Oxford Diffraction, Yarnton, England.

Sakaida, S., Otsubo, K., Sakata, O., Song, C., Fujiwara, A., Takata, M. \& Kitagawa, H. (2016). Nat. Chem. 8, 377-383.

Sciortino, N. F., Scherl-Gruenwald, K. R., Chastanet, G., Halder, G. J., Chapman, K. W., Létard, J.-F. \& Kepert, C. J. (2012). Angew. Chem. Int. Ed. 51, 10154-10158.

Sciortino, N. F., Zenere, K. A., Corrigan, M. E., Halder, G. J., Chastanet, G., Létard, J.-F., Kepert, C. J. \& Neville, S. M. (2017). Chem. Sci. 8, 701-707.

Sheldrick, G. M. (2015a). Acta Cryst. A71, 3-8.

Sheldrick, G. M. (2015b). Acta Cryst. C71, 3-8. 


\section{supporting information}

Acta Cryst. (2019). E75, 1536-1539 [https://doi.org/10.1107/S2056989019012945]

\section{Crystal structure of poly[[diaquatetra- $\mu_{2}$-cyanido-iron(II)platinum(II)] acetone disolvate]}

Iryna S. Kuzevanova, Dina D. Naumova, Kateryna V. Terebilenko, Sergiu Shova and II'ya A.

\section{Gural'skiy}

Computing details

Data collection: CrysAlis PRO (Rigaku OD, 2015); cell refinement: CrysAlis PRO (Rigaku OD, 2015); data reduction: CrysAlis PRO (Rigaku OD, 2015); program(s) used to solve structure: ShelXT (Sheldrick, 2015a); program(s) used to refine structure: SHELXL (Sheldrick, 2015b); molecular graphics: OLEX2 (Dolomanov et al., 2009); software used to prepare material for publication: OLEX2 (Dolomanov et al., 2009).

Poly[[diaquatetra- $\mu_{2}$-cyanido-iron(II)platinum(II)] acetone disolvate]

Crystal data

$\left[\mathrm{FePt}(\mathrm{CN})_{4}\left(\mathrm{H}_{2} \mathrm{O}\right)_{2}\right] \cdot 2 \mathrm{C}_{3} \mathrm{H}_{6} \mathrm{O}$

$M_{r}=507.21$

Tetragonal, $P 4 / \mathrm{mmm}$

$a=7.4802(4) \AA$

$c=7.9725(11) \AA$

$V=446.09(8) \AA^{3}$

$Z=1$

$F(000)=240$

Data collection

Rigaku Oxford Diffraction Xcalibur, Eos diffractometer

Radiation source: fine-focus sealed X-ray tube, Enhance (Mo) X-ray Source

Graphite monochromator

Detector resolution: 8.0797 pixels $\mathrm{mm}^{-1}$

$\omega$ scans

Absorption correction: multi-scan

(CrysAlisPro; Rigaku OD, 2015)

Refinement

Refinement on $F^{2}$

Least-squares matrix: full

$R\left[F^{2}>2 \sigma\left(F^{2}\right)\right]=0.025$

$w R\left(F^{2}\right)=0.046$

$S=1.03$

361 reflections

34 parameters

0 restraints
$D_{\mathrm{x}}=1.888 \mathrm{Mg} \mathrm{m}^{-3}$

Mo $K \alpha$ radiation, $\lambda=0.71073 \AA$

Cell parameters from 664 reflections

$\theta=2.5-28.5^{\circ}$

$\mu=8.66 \mathrm{~mm}^{-1}$

$T=180 \mathrm{~K}$

Plate, clear colourless

$0.05 \times 0.05 \times 0.02 \mathrm{~mm}$

$T_{\min }=0.699, T_{\max }=1.000$

1126 measured reflections

361 independent reflections

359 reflections with $I>2 \sigma(I)$

$R_{\text {int }}=0.037$

$\theta_{\max }=29.0^{\circ}, \theta_{\min }=2.6^{\circ}$

$h=-5 \rightarrow 10$

$k=-10 \rightarrow 5$

$l=-10 \rightarrow 5$

Primary atom site location: dual

Hydrogen site location: mixed

$\mathrm{H}$-atom parameters constrained

$w=1 /\left[\sigma^{2}\left(F_{\mathrm{o}}^{2}\right)+(0.0197 P)^{2}\right]$

where $P=\left(F_{\mathrm{o}}^{2}+2 F_{\mathrm{c}}^{2}\right) / 3$

$(\Delta / \sigma)_{\max }<0.001$

$\Delta \rho_{\max }=1.25 \mathrm{e} \AA^{-3}$

$\Delta \rho_{\min }=-1.06 \mathrm{e}^{-3}$ 


\section{Special details}

Geometry. All esds (except the esd in the dihedral angle between two 1.s. planes) are estimated using the full covariance matrix. The cell esds are taken into account individually in the estimation of esds in distances, angles and torsion angles; correlations between esds in cell parameters are only used when they are defined by crystal symmetry. An approximate (isotropic) treatment of cell esds is used for estimating esds involving l.s. planes.

Fractional atomic coordinates and isotropic or equivalent isotropic displacement parameters $\left(\AA^{2}\right)$

\begin{tabular}{llllll}
\hline & $x$ & $y$ & $z$ & $U_{\text {iss }} / U_{\text {eq }}$ & Occ. $(<1)$ \\
\hline Pt1 & 0.500000 & 0.500000 & 0.500000 & $0.01400(17)$ & \\
Fe1 & 0.000000 & 0.000000 & 0.500000 & $0.0104(4)$ & \\
O1 & 0.000000 & 0.000000 & $0.2329(8)$ & $0.0276(16)$ & \\
H1A & 0.105309 & 0.000432 & 0.196819 & $0.041^{*}$ & 0.25 \\
H1B & -0.043425 & 0.098216 & 0.196839 & $0.041^{*}$ & 0.125 \\
C1 & $0.3116(6)$ & $0.3116(6)$ & 0.500000 & $0.0186(15)$ & \\
N1 & $0.2040(5)$ & $0.2040(5)$ & 0.500000 & $0.0210(13)$ & \\
C3 & $0.475(9)$ & $0.036(8)$ & $0.1546(18)$ & $0.08(2)$ & 0.25 \\
H3A & 0.473348 & 0.162844 & 0.175407 & $0.114^{*}$ & 0.25 \\
H3B & 0.531096 & -0.023709 & 0.246770 & $0.114^{*}$ & 0.25 \\
H3C & 0.354004 & -0.005924 & 0.142949 & $0.114 *$ & 0.25 \\
O2 & $0.7276(18)$ & $0.043(3)$ & 0.000000 & $0.055(5)$ & 0.25 \\
C2 & $0.574(2)$ & 0.000000 & 0.000000 & $0.055(5)$ & 0.5 \\
\hline
\end{tabular}

Atomic displacement parameters $\left(\AA^{2}\right)$

\begin{tabular}{lllllll}
\hline & $U^{11}$ & $U^{22}$ & $U^{33}$ & $U^{12}$ & $U^{13}$ & $U^{23}$ \\
\hline Pt1 & $0.00585(17)$ & $0.00585(17)$ & $0.0303(3)$ & 0.000 & 0.000 & 0.000 \\
Fe1 & $0.0068(5)$ & $0.0068(5)$ & $0.0177(10)$ & 0.000 & 0.000 & 0.000 \\
O1 & $0.030(2)$ & $0.030(2)$ & $0.023(4)$ & 0.000 & 0.000 & 0.000 \\
C1 & $0.0092(18)$ & $0.0092(18)$ & $0.037(4)$ & $0.003(2)$ & 0.000 & 0.000 \\
N1 & $0.0121(16)$ & $0.0121(16)$ & $0.039(4)$ & $-0.005(2)$ & 0.000 & 0.000 \\
C3 & $0.04(4)$ & $0.13(5)$ & $0.056(9)$ & $-0.03(2)$ & $0.013(14)$ & $-0.05(2)$ \\
O2 & $0.019(4)$ & $0.109(15)$ & $0.036(6)$ & $-0.005(10)$ & 0.000 & 0.000 \\
C2 & $0.019(4)$ & $0.109(15)$ & $0.036(6)$ & $-0.005(10)$ & 0.000 & 0.000 \\
\hline
\end{tabular}

Geometric parameters $\left(\AA,{ }^{\circ}\right)$

\begin{tabular}{llll}
\hline $\mathrm{Pt} 1-\mathrm{C} 1^{\mathrm{i}}$ & $1.993(6)$ & $\mathrm{Fe} 1-\mathrm{N} 1^{\mathrm{v}}$ & $2.158(5)$ \\
$\mathrm{Pt} 1-\mathrm{C} 1$ & $1.993(6)$ & $\mathrm{Fe} 1-\mathrm{N} 1^{\mathrm{vi}}$ & $2.158(5)$ \\
$\mathrm{Pt} 1-\mathrm{C} 1^{\mathrm{ii}}$ & $1.993(6)$ & $\mathrm{Fe} 1-\mathrm{N} 1$ & $2.158(5)$ \\
$\mathrm{Pt} 1-\mathrm{C} 1^{\mathrm{iii}}$ & $1.993(6)$ & $\mathrm{C} 1-\mathrm{N} 1$ & $1.138(8)$ \\
$\mathrm{Fe} 1-\mathrm{O} 1^{\mathrm{iv}}$ & $2.130(6)$ & $\mathrm{C} 3-\mathrm{C} 2$ & $1.46(4)$ \\
$\mathrm{Fe} 1-\mathrm{O} 1$ & $2.130(6)$ & $\mathrm{O} 2-\mathrm{O} 2^{\mathrm{vi}}$ & $0.64(5)$ \\
$\mathrm{Fe} 1-\mathrm{N} 1^{\mathrm{iv}}$ & $2.158(5)$ & $\mathrm{O} 2-\mathrm{C} 2$ & $1.19(2)$ \\
& & & 90.0 \\
$\mathrm{C} 1^{\mathrm{i}}-\mathrm{Pt} 1-\mathrm{C} 1^{\mathrm{ii}}$ & 90.0 & $\mathrm{O} 1^{\mathrm{i}}-\mathrm{Fe} 1-\mathrm{N} 1^{\mathrm{vi}}$ & 90.0 \\
$\mathrm{C} 1^{\mathrm{ii}}-\mathrm{Pt} 1-\mathrm{C} 1$ & 90.0 & $\mathrm{~N} 1^{\mathrm{iv}}-\mathrm{Fe} 1-\mathrm{N} 1^{\mathrm{vi}}$ & 90.0 \\
$\mathrm{C} 1^{\mathrm{i}}-\mathrm{Pt} 1-\mathrm{C} 1$ & 180.0 & $\mathrm{~N} 1-\mathrm{Fe} 1-\mathrm{N} 1^{\mathrm{vi}}$ &
\end{tabular}




\begin{tabular}{|c|c|c|c|}
\hline $\mathrm{C} 1^{\mathrm{i}}-\mathrm{Pt} 1-\mathrm{C} 1^{\mathrm{iii}}$ & 90.0 & $\mathrm{~N} 1^{\mathrm{iv}}-\mathrm{Fe} 1-\mathrm{N} 1^{\mathrm{v}}$ & 90.0 \\
\hline $\mathrm{C}{ }^{\mathrm{ii}}-\mathrm{Pt} 1-\mathrm{C} 1$ & 90.0 & $\mathrm{~N} 1-\mathrm{Fe} 1-\mathrm{N} 1^{\mathrm{iv}}$ & 180.0 \\
\hline $\mathrm{C} 1^{\mathrm{ii}}-\mathrm{Pt} 1-\mathrm{C} 1^{\mathrm{iii}}$ & 180.0 & $\mathrm{~N} 1-\mathrm{Fe} 1-\mathrm{N} 1^{\mathrm{v}}$ & 90.0 \\
\hline $\mathrm{O} 1-\mathrm{Fe} 1-\mathrm{O}^{\mathrm{iv}}$ & 180.0 & 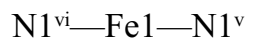 & 180.0 \\
\hline $\mathrm{O} 1-\mathrm{Fe} 1-\mathrm{N} 1$ & 90.0 & $\mathrm{~N} 1-\mathrm{C} 1-\mathrm{Pt} 1$ & 180.0 \\
\hline $\mathrm{O} 1-\mathrm{Fe} 1-\mathrm{N} 1^{\mathrm{vi}}$ & 90.0 & $\mathrm{C} 1-\mathrm{N} 1-\mathrm{Fe} 1$ & 180.0 \\
\hline $\mathrm{O} 1^{\mathrm{iv}}-\mathrm{Fe} 1-\mathrm{N} 1^{\mathrm{v}}$ & 90.0 & $\mathrm{O} 2{ }^{\mathrm{vii}}-\mathrm{O} 2-\mathrm{C} 2$ & $74.4(12)$ \\
\hline $\mathrm{O}^{\mathrm{iv}}-\mathrm{Fe} 1-\mathrm{N} 1$ & 90.0 & $\mathrm{O} 2^{\mathrm{vii}}-\mathrm{C} 2-\mathrm{C} 3$ & $123(2)$ \\
\hline $\mathrm{O} 1-\mathrm{Fe} 1-\mathrm{N} 1^{\mathrm{v}}$ & 90.0 & $\mathrm{O} 2-\mathrm{C} 2-\mathrm{C} 3$ & $116(2)$ \\
\hline $\mathrm{O} 1-\mathrm{Fe} 1-\mathrm{N} 1^{\mathrm{iv}}$ & 90.0 & $\mathrm{O} 2-\mathrm{C} 2-\mathrm{O} 2^{\mathrm{vii}}$ & $31(2)$ \\
\hline $\mathrm{O} 1^{\mathrm{iv}}-\mathrm{Fe} 1-\mathrm{N} 1^{\mathrm{iv}}$ & 90.0 & & \\
\hline
\end{tabular}

Symmetry codes: (i) $-x+1,-y+1,-z+1$; (ii) $y,-x+1,-z+1$; (iii) $-y+1, x, z$; (iv) $-x,-y,-z+1$; (v) $-y, x, z$; (vi) $y,-x,-z+1$; (vii) $x,-y, z$.

Hydrogen-bond geometry $\left(A,{ }^{\circ}\right)$

\begin{tabular}{lllll}
\hline$D-\mathrm{H} \cdots A$ & $D-\mathrm{H}$ & $\mathrm{H} \cdots A$ & $D \cdots A$ & $D-\mathrm{H} \cdots A$ \\
\hline $\mathrm{O} 1-\mathrm{H} 1 A \cdots \mathrm{O} 2^{\text {viii }}$ & 0.84 & 2.03 & $2.775(11)$ & 147 \\
$\mathrm{O} 1-\mathrm{H} 1 A \cdots \mathrm{O} 2^{\text {ix }}$ & 0.84 & 2.03 & $2.775(11)$ & 148 \\
$\mathrm{O} 1-\mathrm{H} 1 B \cdots \mathrm{O} 2^{\mathrm{x}}$ & 0.85 & 2.04 & $2.775(11)$ & 144 \\
$\mathrm{O} 1-\mathrm{H} 1 B \cdots \mathrm{O} 2^{\mathrm{xi}}$ & 0.85 & 2.14 & $2.775(11)$ & 131
\end{tabular}

Symmetry codes: (viii) $-x+1,-y, z$; (ix) $-x+1, y,-z$; (x) $-y,-x+1,-z$; (xi) $y,-x+1, z$. 